\title{
Overexpression of ASPH protein predicts poor outcomes in retroperitoneal liposarcoma patients
}

\section{Mengmeng Xiao}

Peking University International Hospital https://orcid.org/0000-0003-2309-9480

\section{Xiaobing Chen}

Peking University International Hospital

\section{Yuqing Cui}

Tumor Hospital of Harbin Medical University

\section{Lihua Wang}

Peking University International Hospital

\section{Xiaosong Rao}

Peking University International Hospital

\section{Xuesong Chen}

Tumor Hospital of Harbin Medical University

Chenghua Luo ( $\sim$ pkuihlch@163.com )

https://orcid.org/0000-0002-5939-7888

\section{Research article}

Keywords: Liposarcoma; Retroperitoneal tumor; Aspartate- $\beta$-hydroxylase (ASPH)

Posted Date: June 10th, 2020

DOI: https://doi.org/10.21203/rs.3.rs-30976/v1

License: (c) (i) This work is licensed under a Creative Commons Attribution 4.0 International License.

Read Full License 


\title{
Overexpression of ASPH protein predicts poor outcomes in
}

\author{
retroperitoneal liposarcoma patients \\ Mengmeng Xiao ${ }^{1,2 \#}$, Xiaobing Chen ${ }^{1,2 \#}$, Yuqing Cui ${ }^{3}$, Lihua Wang ${ }^{2,4}$, Xiaosong $\mathrm{Rao}^{2,4}$, \\ Xuesong Chen $^{3}$, Chenghua Luo ${ }^{1,2^{*}}$
}

1. Department of Retroperitoneal Tumor Surgery, Peking University International Hospital, Beijing, China

2. The Eighth Affiliated Hospital of Peking University Health Science Center, Beijing, China

3. Department of Oncology, Cancer Hospital Affiliated to Harbin Medical University, Harbin, China

4. Department of Pathology, Peking University International Hospital, Beijing, China

\section{\# Mengmeng Xiao \& Xiaobing Chen contributed equally to this work.}

*To whom correspondence should be addressed:

Chenghua Luo, MD, PhD, Department of Retroperitoneal Tumor Surgery, Peking

University International Hospital, The Eighth Affiliated Hospital of Peking University Health Science Center, Beijing 102206, P. R. China,

E-mail: pkuihlch@163.com

Key words: Liposarcoma; Retroperitoneal tumor; Aspartate- $\beta$-hydroxylase (ASPH) 


\begin{abstract}
Background: No druggable targets and prognostic factors are currently available for retroperitoneal liposarcoma (RPLS) due to very limited understanding of its molecular mechanisms and pathogenesis. This study aims to decipher expression profiling and prognostic value of aspartate- $\beta$-hydroxylase (ASPH), an activator of Notch signaling pathway, in RPLS.
\end{abstract}

Methods: Totally 138 patients with RPLS who received resection were recruited in this retrospective study. Immunohistochemistry was performed to decipher expression profiling of ASPH in archived specimens. Prognostic value of ASPH was evaluated by Kaplan-Meier plot and Cox proportional hazards model. Chi-square test was used to compare counting data.

Results: The overall positive rate of ASPH expression in RPLS was $90.6 \%$. Well-differentiated liposarcoma had a similar positive rate to dedifferentiated liposarcoma (90.2\% vs. 91.1\%). A high level of ASPH expression correlated with reduced postoperative recurrence-free survival rate and overall survival rate $(\mathrm{P}<0.05)$.

Conclusions: ASPH is an independent predictor for clinical outcome of patients with RPLS. A high level of ASPH expression confers poor post-operational prognosis. 


\section{Background}

Liposarcoma is a common subtype of retroperitoneal sarcoma [1]. Due to the complicated anatomical heterogeneity of retroperitoneal liposarcoma (RPLS), either adjacent to or even invading into major vessels and important organs when diagnosed, massive bleeding frequently occurs during surgery. Thus, combined viscera resection is required, which makes the operation on RPLS much more challenging than other solid tumors, resulting in a much lower resection rate [2]. Currently, surgery is the most effective treatment for RPLS, and adjuvant therapies have shown little/no efficacy [3,4]. Chemotherapy has no definite therapeutic effect on RPLS [5], and radiotherapy is very limited due to serious toxicity to adjacent abdominal organs [6]. There is a paucity of research focusing specifically on molecular mechanisms underlying RPLS pathogenesis. Consequently, no diagnostic biomarkers, druggable targets or prognostic factors are available for RPLS.

Aspartate- $\beta$-hydroxylase (aspartyl- $\beta$-hydroxylase or asparaginyl- $\beta$-hydroxylase; ASPH) is highly conservative deoxidizing enzyme [7]. The ASPH is critical for embryonic development. Normally, ASPH is silenced during adulthood and only expressed in placenta [8,9]. Abnormal re-expression/upregulation of ASPH occurs in vast majority of malignancies, such as hepatocellular carcinoma, lung cancer, colorectal cancer, breast cancer and pancreatic cancer [10-12].

The enzymatic activity of ASPH depends on its catalytic domain [13,14], which hydroxylates aspartic acid or asparagine residues in EGF-like repeats of several proteins (such as Notch receptors and Notch ligands) in the presence of iron divalent, thus mediating cellular motility and differentiation $[15,14,13]$. Previously, correlation between ASPH mRNA transcription levels and insulin receptor substrates, growth factors, IGF receptors, Notch, Jagged, and HES was analyzed. Activation of 
IGF-1/IGF-2 signal leads to upregulation of ASPH and thus Notch. Biologically, ASPH promotes tumorigenesis partially through activating Notch and SRC signaling pathways [16,17]. Notably, ASPH's oncogenic properties rely on its $\beta$-hydroxylase activity, which enhances proliferation, migration, invasion, and metastasis $[18,19]$.

The Notch signaling pathway is indicated to be highly activated in RPLS, and the growth of liposarcoma cells can be significantly blunted by knocking-down of Notch [20]. Hence, we explored if ASPH is associated with tumor characteristics and clinical outcome of RPLS. Here, in a large RPLS cohort, we performed immunohistochemistry to detect the expression profiling of ASPH and evaluate its potential in prognostics of RPLS.

\section{Methods}

\section{Patient selection}

After the ethical approved, 179 patients with RPLS were admitted to Department of retroperitoneal tumor surgery, Peking University International Hospital, from December 1, 2014 to February 28, 2018. Among them, 24 cases had surgical residues, 5 cases died due to serious postoperative complications, and 12 cases had extremely rare subtypes. These 41 cases were excluded from data analysis. Thus, 138 cases (71 males and 67 females) were eventually included in this study. All cases were confirmed by histopathologic and immunohistochemical examination. The mean age of patient population was $54.6 \pm 11.5$. Histologically, 82 cases had well-differentiated liposarcoma (WD) and 56 had dedifferentiated liposarcoma (DD). All patients received no chemoradiotherapy or targeted therapy before surgery. The general information and FNCLCC scores of these patients were retrospectively collected from electronic medical record system. 


\section{Immunohistochemistry}

Formalin fixation and paraffin-embedded (FFPE) tissue blocks from archived tissue-bank were applied for immunohistochemical staining. In brief, specimens were sectioned at $4 \mu \mathrm{m}$-thick, deparaffinized in xylene, and rehydrated, followed by antigen retrieval in sodium citrate. Then slides were incubated with primary monoclonal antibody against ASPH (1:4000 dilution, courtesy of Professor Jack R. Wands, Brown University, USA) overnight at $4^{\circ} \mathrm{C}$. Afterwards, biotin-labeled goat anti-mouse secondary antibody (Beijing Zhong Shan Jin Qiao biotechnology co. LTD) was added. Sections were then counterstained with hematoxylin, dehydrated, and mounted.

Immunohistochemical staining was evaluated and scored by 3 independent pathologists. Four high-power fields $(\times 200)$ were randomly selected for each slide, and the number of positive cells in a single field was counted and scored according to staining distribution: score 0 (negative), score 1 (0-25\%), score 2 (26\%-50\%), score 3 (51\%-100\%). The average score of all fields was calculated. The average score $\leq 0.5$ was set as negative to low expression, and $>0.5$ as moderate to high expression.

\section{Follow-up}

All cases were followed up by telephone or outpatient. The follow-up period was from the patient's discharge to October 2019 or the death date. Median follow-up was 21.5 months (4-53 months). Among the 138 patients, 84 relapsed, 43 died (all due to tumor recurrence and metastasis), and 14 were out of contact. The follow-up rate was $89.8 \%$.

\section{Statistical analysis}

Statistical software SPSS19.0 was used to analyze the data. Chi-square test was used for the analysis of counting data. The Kaplan-Meier method was used for survival analysis. COX regression model was used for the correlation analysis. 
Comparisons made in which $\mathrm{P}<0.05$ were considered statistically significant.

\section{Results}

\section{Expression of ASPH in RPLS}

Compared to adjacent normal tissue, ASPH was overexpressed in RPLS tumor in most cases (Figure 1). The overall positive rate of ASPH staining was $90.6 \%$. The positive rate $[90.2 \%(74 / 82)]$ in well-differentiated RPLS was significantly lower than that in dedifferentiated RPLS [91.1\% $(51 / 56), p=0.028]$.

\section{Associations between ASPH expression and tumor characteristics of RPLS}

There was no difference for ASPH expression between patients divided by age, gender, tumor number, P53 expression, and MDM2 expression ( $\mathrm{P}>0.05$, Table 1). The patients with worse pathology subtypes $(\mathrm{P}=0.028)$ and higher FNCLCC grades $(\mathrm{P}=0.007)$ showed a higher ASPH expression level than others (Table 1).

\section{ASPH is an independent prognostic factor for RPLS}

Kaplan-Meier analysis showed that the overall survival (OS) of patients with high ASPH expression was much worse than that of patients with low expression (Figure 2A, $\chi^{2}=6.56, \mathrm{p}=0.010$ ). The relapse-free survival (RFS) of patients with high ASPH expression was also much worse than that of patients with low ASPH expression (Figure $2 \mathrm{~B}, \chi^{2}=7.17, \mathrm{p}=0.007$ ).

In Cox regression univariate model, expression level of ASPH and FNCLCC grade were identified as prognostic factors $(p<0.05$, Table 2$)$. In multivariate model, FNCLCC grade and expression level of ASPH were independent risk factors of RFS. Compared with lower expression level, the recurrence risk was 1.84 times higher in patients with higher expression level of ASPH $(\mathrm{OR}=1.84,95 \% \mathrm{CI}: 1.12-3.04, \mathrm{p}=0.017$, Table 2). 


\section{Discussion}

Compared with well-differentiated RPLS, dedifferentiated RPLS is much more malignant [21], with unfavorable histopathological and clinical characters such as invasion into adjacent tissues at an early stage and a higher FNCLCC grade [22]. In this study, ASPH expression in dedifferentiated RPLS was significantly higher than that in well-differentiated RPLS, and related to FNCLCC grade. Thus, ASPH may play a role in the progression of RPLS, especially in dedifferentiated RPLS.

Importantly, ASPH was an independent risk factor for relapse of RPLS. Patients with a higher level of ASPH expression were more likely to relapse. The effect of ASPH expression on OS is similar to RFS, except an intersection of curves in the period of 46 months after surgery. Considering the complexity and heterogeneity of RPLS patients' preoperative status, the unexpected intersection would be possibly explained by complicacy after surgery. Some patients with RPLS may survive a relatively long time through receiving repeated or even multiple operations. Some patients give up further treatment after suffering from economic difficulties, psychological fear and complications after multiple surgical operations. All those factors make OS a less objective indicator than RFS.

It has been reported that ASPH promotes proliferation, migration, invasion in various cancer types, including hepatocellular carcinoma [23], breast carcinoma [24] and pancreatic cancer. Thus, ASPH acts as an oncogene and is expected to enhance aggressive/malignant cellular behaviors, possibly leading to a poor prognosis in RPLS. This hypothesis is under evaluation by cytological experiments. In this study, IHC data have provided supporting evidence for ASPH's oncogenic role in RPLS.

Previous studies have demonstrated that clinicopathological factors such as pathological subtypes and tumor size are associated with prognosis [25]. Some studies 
have suggested that S-100, Ki-67 [26], MDM2 and P53 [27] are associated with the prognosis of RPLS. However, with a much larger cohort, neither MDM2 nor P53 is a prognostic factor. Instead, ASPH has been identified as a potential prognostic factor in RPLS. Considering the embryonic origins of RPLS are different from cancers originated from epithelium, very few tumor biomarkers are valid in RPLS. However, before a better understanding of molecular mechanisms of RPLS, ASPH with pan-cancerous verification would be the first choice in development of RPLS biomarkers.

The biological characteristics of RPLS are different from carcinomas. The RPLS tend to grow expansively and is prone to postoperative recurrence, but rarely exhibits distant metastasis [27]. Thus, RPLS patients receive repeated and multiple surgical treatment. It is extremely important for such patients to prolong overall/disease-free survival without recurrence after surgery, and to gain "time" and "space" for the next surgical resection. Our results suggested that patients with high-ASPH RPLS would be considered as high-risk of relapse, which should receive a much closer observation and longer follow-up.

It is reported that inhibiting expression or enzymatic activity of ASPH could undermine proliferation, migration, invasion, and metastasis of various tumor cells $[28,29]$. In addition, ASPH can serve as an immunotherapy target for liver cancer [30]. Further studies are needed to verify histopathological effects of ASPH on RPLS tumor cells, which would help to determine if ASPH can be used as a druggable target or an immunotherapeutic target for (neo) adjuvant treatment of RPLS.

\section{Conclusions}

In summary, ASPH is an independent predictor for clinical outcome of patients 
with RPLS. A high level of ASPH expression confers poor post-operational prognosis. In particular, our study suggests that ASPH maybe a potential therapeutic target for of RPLS.

\author{
Abbreviations \\ RPLS: retroperitoneal liposarcoma \\ ASPH: aspartate- $\beta$-hydroxylase \\ OS: overall survival \\ RFS: relapse-free survival
}

\title{
Declarations
}

\section{Ethics approval and consent to participate}

This study was approved by the Ethics committee of Peking University International Hospital (Beijing, China) and all participants signed informed consent forms.

\section{Availability of data and material}

The datasets used and/or analysed during the current study available from the corresponding author on reasonable request.

\section{Consent for publication}

Not applicable

\section{Competing interests}

The authors declare that they have no competing interests.

\section{Funding}

This study was funded by Peking University International Hospital Research Grant (YN2019QN11); Beijing science and technology commission "Applied 
research on clinical characteristics of the capital city" (Z171100001017095).

\section{Authors' contributions}

MMX and XBC carried out the main analysis. CHL conceived and designed the study. YQC, LHW, and XSR helped to collect and reformat the primary data. XSC helped to analyze data and revise the manuscript. MMX and XBC draft the manuscript. All authors have read and approved the manuscript.

\section{Acknowledgements}

Not Applicable

\section{References:}

1Windham TC, Pisters PW. Retroperitoneal sarcomas. CANCER CONTROL. 2005;12(1):36-43. 'doi:'10.1177/107327480501200105.

2Lee SY, Goh BK, Teo MC, Chew MH, Chow PK, Wong WK et al. Retroperitoneal liposarcomas: the experience of a tertiary Asian center. WORLD J SURG ONCOL. 2011;9:12. 'doi:'10.1186/1477-7819-9-12.

3Erzen D, Sencar M, Novak J. Retroperitoneal sarcoma: 25 years of experience with aggressive surgical treatment at the Institute of Oncology, Ljubljana. J SURG ONCOL. 2005;91(1):1-9. 'doi:'10.1002/jso.20265.

4Alldinger I, Yang Q, Pilarsky C, Saeger HD, Knoefel WT, Peiper M. Retroperitoneal soft tissue sarcomas: prognosis and treatment of primary and recurrent disease in 117 patients. ANTICANCER RES. 2006;26(2B):1577-81

5Jones RL, Fisher C, Al-Muderis O, Judson IR. Differential sensitivity of liposarcoma subtypes to chemotherapy. EUR J CANCER. 2005;41(18):2853-60

6Pawlik TM, Pisters PWT, Mikula L, Feig BW, Hunt KK, Cormier JN et al. Long-Term Results of Two Prospective Trials of Preoperative External Beam Radiotherapy for Localized Intermediate- or High-Grade Retroperitoneal Soft Tissue Sarcoma. ANN SURG ONCOL. 2006;13(4):508-17

7Ince N, Monte SMDL, Wands JR. Overexpression of Human Aspartyl (Asparaginyl) $\beta$ -Hydroxylase Is Associated with Malignant Transformation. CANCER RES. 2000;60(5):1261-6

8Patel N, Khan AO, Mansour A, Mohamed JY, Al-Assiri A, Haddad R et al. Mutations in ASPH Cause Facial Dysmorphism, Lens Dislocation, Anterior-Segment Abnormalities, and Spontaneous Filtering Blebs, or Traboulsi Syndrome. AM J HUM GENET. 2014;94(5):755-9

9Lavaissiere L, Jia S, Nishiyama M, Monte SDL, Friedman PA. Overexpression of human aspartyl(asparaginyl) $\beta$-hydroxylase in hepatocellular carcinoma and cholangiocarcinoma. J CLIN INVEST. 1996;98(6):1313-23

10Xue T, Xue X, Huang Q, Wei L, Sun K, Xue T. Monoclonal Antibodies Against Human Aspartyl (Asparaginyl) beta-Hydroxylase Developed by DNA Immunization. Hybridoma. 2009;28(4):251-7 
11Nagaoka K, Bai X, Ogawa K, Dong X, Zhang S, Zhou Y et al. Anti-tumor activity of antibody drug conjugate targeting aspartate-beta-hydroxylase in pancreatic ductal adenocarcinoma. CANCER LETT. 2019;449:87-98. 'doi:'10.1016/j.canlet.2019.02.006.

12Lin Q, Chen X, Meng F, Ogawa K, Li M, Song R et al. ASPH-notch Axis guided Exosomal delivery of Prometastatic Secretome renders breast Cancer multi-organ metastasis. MOL CANCER. 2019;18(1):156. 'doi:'10.1186/s12943-019-1077-0.

13 Treves S, Feriotto G, Moccagatta L, Gambari R, Zorzato F. Molecular Cloning, Expression, Functional Characterization, Chromosomal Localization, and Gene Structure of Junctate, a Novel Integral Calcium Binding Protein of Sarco(endo)plasmic Reticulum Membrane. J BIOL CHEM. 2000;275(50):39555-68

14Dinchuk JE, Henderson NL, Burn TC, Huber R, Ho SP, Link J et al. Aspartyl beta -hydroxylase (Asph) and an evolutionarily conserved isoform of Asph missing the catalytic domain share exons with junctin. J BIOL CHEM. 2000;275(50):39543-54. 'doi:'10.1074/jbc.M006753200.

15Dinchuk JE, Focht RJ, Kelley JA, Henderson NL, Zolotarjova NI, Wynn R et al. Absence of post-translational aspartyl beta-hydroxylation of epidermal growth factor domains in mice leads to developmental defects and an increased incidence of intestinal neoplasia. J BIOL CHEM. 2002;277(15):12970-7. 'doi:'10.1074/jbc.M110389200.

16Cantarini MC, Sm DLM, Pang M, Tong M, D'Errico A, Trevisani F et al. Aspartyl-asparagyl beta hydroxylase over-expression in human hepatoma is linked to activation of insulin-like growth factor and notch signaling mechanisms. HEPATOLOGY. 2010;44(2):446-57

17Ogawa K, Lin Q, Li L, Bai X, Chen X, Chen H et al. Aspartate beta-hydroxylase promotes pancreatic ductal adenocarcinoma metastasis through activation of SRC signaling pathway. J HEMATOL ONCOL. 2019;12(1):144. 'doi:'10.1186/s13045-019-0837-z.

18Dinchuk JE, Focht RJ, Kelley JA, Henderson NL, Zolotarjova NI, Richard W et al. Absence of post-translational aspartyl beta-hydroxylation of epidermal growth factor domains in mice leads to developmental defects and an increased incidence of intestinal neoplasia. J BIOL CHEM. 2002;277(15):12970-7

19Ogawa K, Lin Q, Li L, Bai X, Chen X, Chen H et al. Prometastatic Secretome Trafficking via Exosomes Initiates Pancreatic Cancer Pulmonary Metastasis. CANCER LETT. 2020. 'doi:'10.1016/j.canlet.2020.02.039.

20Meng RD, Qin L, Shelton CC, Li Y, Maki RG, Brill ER et al. Association of Notch signaling pathway expression in liposarcomas with outcome, and targeting with gamma-secretase inhibitors. J CLIN ONCOL. 2009

21Qiu F, Xu C, Miao C. Retroperitoneal Liposarcoma. In: Luo C, 'editor'. Retroperitoneal Tumors: Clinical Management. Dordrecht: Springer Netherlands; 2018. p. 153-61.

22Mussi C, Collini P, Miceli R, Barisella M, Mariani L, Fiore M et al. The prognostic impact of dedifferentiation in retroperitoneal liposarcoma: a series of surgically treated patients at a single institution. CANCER-AM CANCER SOC. 2008;113(7):1657-65. 'doi:'10.1002/cncr.23774.

23Zou Q, Hou Y, Wang H, Wang K, Xing X, Xia Y et al. Hydroxylase Activity of ASPH Promotes Hepatocellular Carcinoma Metastasis Through Epithelial-to-Mesenchymal Transition Pathway. EBIOMEDICINE. 2018;31:287-98

24Shimoda M, Hori A, Wands JR, Tsunashima R, Naoi Y, Miyake T et al. Endocrine sensitivity of estrogen receptor - positive breast cancer is negatively correlated with aspartate - $\beta$ - hydroxylase expression. CANCER SCI. 2017;108(12) 
25Singer S, Antonescu CR, Riedel E, Brennan MF. Histologic subtype and margin of resection predict pattern of recurrence and survival for retroperitoneal liposarcoma. ANN SURG. 2003;238(3):358

26Wu YX, Liu JY, Liu JJ, Yan P, Tang B, Cui YH et al. A retrospective, single-center cohort study on 65 patients with primary retroperitoneal liposarcoma. ONCOL LETT. 2018;15(2):1799-810

27Wang YL, Qiu JS. Relationship between Expression of c-myc and p53 in Liposarcoma. CHIN J CANCER. 2002;21(1):63-7

28Aihara A, Huang CK, Olsen MJ, Lin Q, Chung W, Tang Q et al. A cell-surface beta-hydroxylase is a biomarker and therapeutic target for hepatocellular carcinoma. HEPATOLOGY. 2014;60(4):1302-13. 'doi:'10.1002/hep.27275.

29Maeda T, Sepe P, Lahousse S, Tamaki S, Enjoji M, Wands JR et al. Antisense oligodeoxynucleotides directed against aspartyl (asparaginyl) beta-hydroxylase suppress migration of cholangiocarcinoma cells. J HEPATOL. 2003;38(5):615-22

30Tomimaru Y, Mishra S, Safran H, Charpentier KP, Martin W, De Groot AS et al. Aspartate- $\beta$ -hydroxylase induces epitope-specific $\mathrm{T}$ cell responses in hepatocellular carcinoma. VACCINE. 2015;33(10):1256-66

\section{Figure Legend}

Figure 1. Immunohistochemical staining for ASPH expression in retroperitoneal liposarcoma tissues.

(A-B) Negative staining of ASPH $(100 \times, 200 \times$, respectively); (C-D) Weak staining of ASPH where scored $=1(100 \times, 200 \times$, respectively, $0-25 \%$ positive cells $) ;(E-F)$ Moderate staining of ASPH where scored $=2(100 \times, 200 \times$, respectively, $25-50 \%$ positive cells); (G-H) Strong staining of ASPH where scored $=3(100 \times, 200 \times$, respectively, $50-100 \%$ positive cells).

Figure 2. OS and RFS of retroperitoneal liposarcoma stratified by ASPH expression levels.

The OS stratified by ASPH expression levels. B. RFS stratified by ASPH expression levels. 
Figures

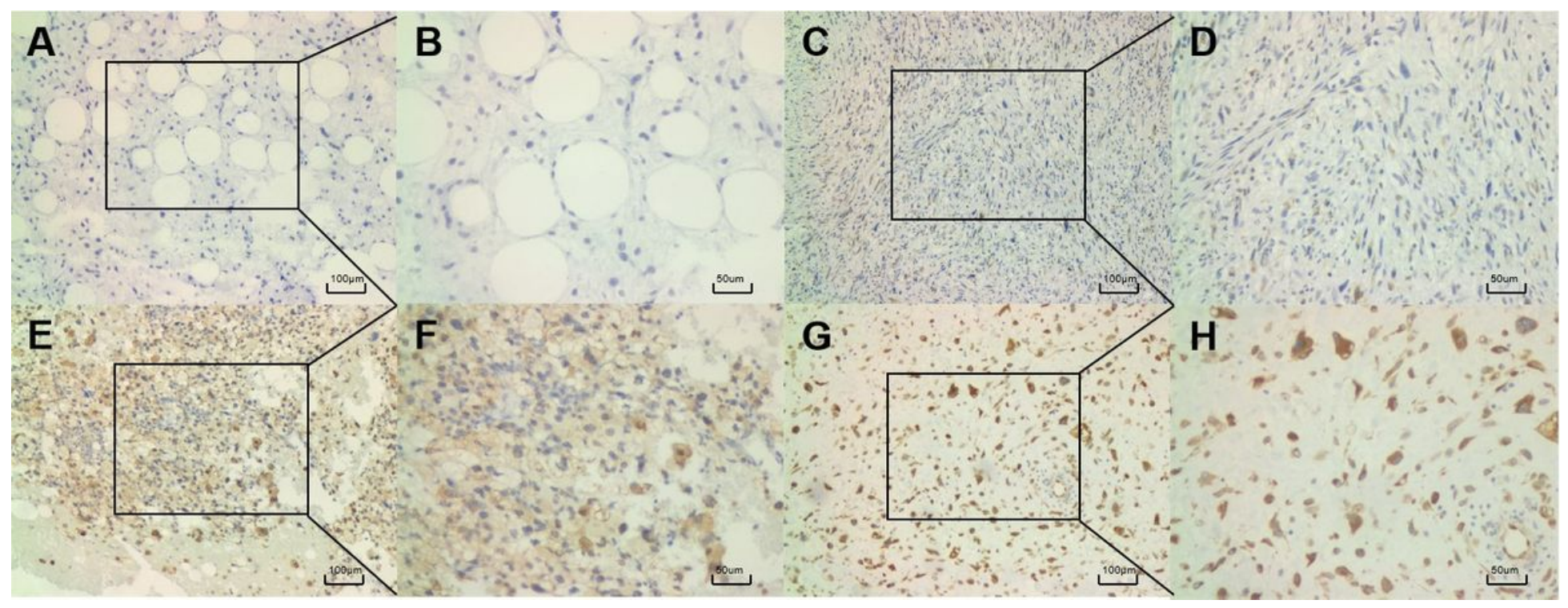

\section{Figure 1}

Immunohistochemical staining for ASPH expression in retroperitoneal liposarcoma tissues. (A-B) Negative staining of ASPH (100x, 200x, respectively); (C-D) Weak staining of ASPH where scored $=1$ (100x, 200x, respectively, 0-25\% positive cells); (E-F) Moderate staining of ASPH where scored = 2 (100x, $200 \times$, respectively, 25-50\% positive cells); (G-H) Strong staining of ASPH where scored $=3$ (100x, 200x, respectively, 50-100\% positive cells).
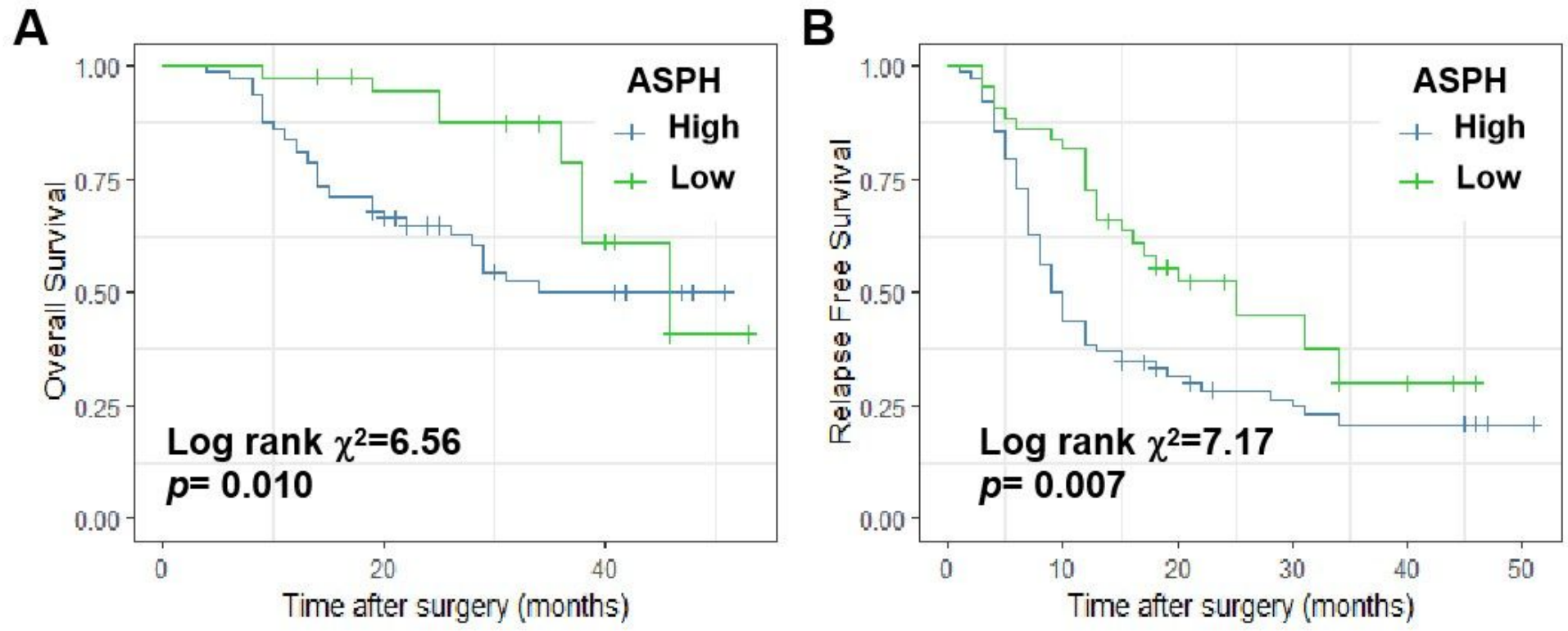

\section{Figure 2}

OS and RFS of retroperitoneal liposarcoma stratified by ASPH expression levels. The OS stratified by ASPH expression levels. B. RFS stratified by ASPH expression levels. 


\section{Supplementary Files}

This is a list of supplementary files associated with this preprint. Click to download.

- Table1.pdf

- Table2.pdf 